\title{
Description of the male of Mangora brokopondo (Araneae, Araneidae), with notes on Mangora species from Brazilian Oriental Amazon
}

\author{
Regiane Saturnino ${ }^{1}$, Emanuel G. Cafofo ${ }^{1}$, Manoel B. A. Neto ${ }^{1}$, Janael Ricetti ${ }^{2}$ \\ \& Alexandre B. Bonaldo ${ }^{1}$
}

1. Museu Paraense Emílio Goeldi, Coordenação de Zoologia, Laboratório de Aracnologia, Av. Perimetral, 1901, Terra Firme, 66077-830, Belém, Pará, Brazil. (sf.regiane@gmail.com,
emanuelgcs@gmail.com, aguianeto7@gmail.com, bonaldo@museu-goeldi.br)
2. Universidade Federal do Paraná, Departamento de Zoologia, Caixa Postal 19020,81531-980, Curitiba, Paraná, Brazil. (jricetti@gmail.com)

ABSTRACT. The male of Mangora brokopondo Levi, 2007 is described and illustrated for the first time. Variation in the color pattern of the females is documented. Mangora woytkowskii Levi, 2007 is considered a junior synonym of M. hirtipes (Taczanowski, 1878). New records of $M$. alinahui Levi, 2007 and M. pia Chamberlin \& Ivie, 1936 are presented.

KEYWORDS. Spiders, Arachnida, taxonomy, distribution, Neotropical Region.

RESUMO. Descrição do macho de Mangora brokopondo (Araneae, Araneidae), com notas sobre espécies de Mangora da Amazônia Oriental brasileira. O macho de Mangora brokopondo Levi, 2007 é descrito e ilustrado pela primeira vez. Variação no padrão de coloração da fêmea é documentada. Mangora woytkowskii Levi, 2007 é considerado sinônimo-júnior de M. hirtipes (Taczanowski, 1878). Novos registros de M. alinahui Levi, 2007 e M. pia Chamberlin \& Ivie, 1936 são apresentados.

PALAVRAS-CHAVE. Aranhas, Arachnida, Taxonomia, distribuição, Região Neotropical.

Araneidae is one of the most species-rich spider families, encompassing 170 genera and more than three thousand species distributed globally (PLATNICK, 2013). Unlike the majority of spider families, taxonomic knowledge of Araneidae is well developed, at least in the Nearctic and Neotropical regions, mostly due to the efforts of a single author, Herbert Levi, who treated almost all American genera of Araneidae, producing high quality taxonomic revisions over the last three decades (e.g. LeVI, 1976, 1988, 1995).

The modern revision of Mangora O. PickardCambridge, 1889 was made in two papers, one dealing with the fauna from Mexico and West Indies, by LEVI (2005) with 32 species, and other dealing with the South American species, by LEVI (2007), who presented 142 species, 127 of which were new to science at that time. Currently, the genus presents 186 species (PlatNicK, 2013), taking into account the Central American species and the synonymy here proposed.

According to LeVI (2007), the Amazon region harbors the higher diversity of Mangora species, both in terms of species richness and abundance of specimens, at least in collections. He further noted that some of the species from the Amazon present wide distribution ranges, contrasting with the small known distributions of most species of the genus. Although many areas remain poorly sampled in the Amazon region, the known records of some of the species here studied indeed suggest wide distributions. For example, some of the records of $M$. pia Chamberlin \& Ivie, 1936 depicted below are more than $700 \mathrm{~km}$ apart, indicating this species may occur across most the Amazon basin. Mangora figure as one of the Araneidae genera with more species collected in inventories carried on in the Brazilian Amazon, along with Alpaida O. Pickard-Cambridge, Eustala Simon, and Micrathena Sundevall (e.g. BonALDo et al., 2010). Our data show that specimens of Mangora are common in both primary and secondary forests and are collected mainly by beating vegetation during the day and by nocturnal manual samplings.

In the present contribution we describe for the first time the male of M. brokopondo Levi, 2007, documenting variation in the color pattern of the female. Recent collecting efforts in the state of Pará, Brasil, enable us to extend the known distribution of $M$. alinahui Levi, 2007 and $M$. pia and provide evidence that the male of $M$. hirtipes (Taczanowski, 1878) was first described as $M$. woytkowskii Levi, 2007. We therefore propose a synonymy of these two specific names. Males of $M$. brokopondo were collected in the Caxiuanã National Forest (FLONA Caxiuanã), a locality where some of the females listed by LEVI (2007) were collected. On the other hand, $M$. hirtipes is both more abundant and widely distributed than previously known, occurring in sympatry with $M$. brokopondo at FLONA Caxiuanã, but also occurring in other Pará localities such as Itaituba and Juruti.

\section{MATERIAL AND METHODS}

The specimens examined are deposited in the Museu Paraense Emílio Goeldi (MPEG, curator: A. B. Bonaldo), Belém, Pará, Brazil and the Museu de Ciências e Tecnologia, Pontífícia Universidade Católica do Rio Grande do Sul, Porto Alegre, Rio Grande do Sul, Brazil (MCTP, curator: A. A. Lise). The format of the 
description and palpal terminology follows Levi (2007). The specimen was illustrated using a stereomicroscope with a camera lucida. The images and measurements (in millimeters) were taken using automontage software. Additional information is furnished between [brackets]. The following abbreviations are used: ALE, anterior lateral eyes; AME, anterior median eyes; C, conductor; E, embolus; MA, median apophysis; PLE, posterior lateral eyes; PME, posterior median eyes; TA, terminal apophysis.

\section{Mangora brokopondo Levi, 2007}

(Figs 1-6)

Mangora brokopondo LevI, 2007:50, figs 137-140 ( $Q$ holotype

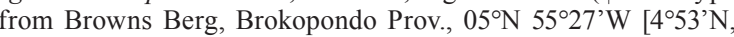
$\left.55^{\circ} 13^{\prime} \mathrm{W}\right]$, Suriname, D. Smith Trail, 20.II.1982, deposited in Museum of Comparative Zoology, not examined).
Diagnosis. Males of $M$. brokopondo resembles those of M. ikuruwa, M. sumauma, M. keduc and $M$. tambo in having a distally bifid embolus (Fig. 2), but differ from those of the first three species by the elongated hyaline conductor (Figs 2, 3) and from those of $M$. tambo by the large, ventrally projected median apophysis (Fig. 2).

Description. Male (MPEG 19085, Melgaço, Pará). Total length 2.5. Carapace length 1.2, width 1.0, height 0.4 . Clypeus height 0.08 . Sternum length 0.5 , width 0.6 . Abdomen length 1.3, width 1.0, height 0.8. Leg formula I/ II-IV/III. Leg lengths: femur, I 1.4, II 1.2, III 0.8, IV 1.3; patella, I 0.4, II 0.4, III 0.3, IV 0.4; tibia, I 1.1, II 0.9, III 0.5, IV 0.9; metatarsus, I 1.2, II 1.1, III 0.8, IV 1.1; tarsus, I 0.5, II 0.5, III 0.3, IV 0.4. Eyes diameters and interdistances:
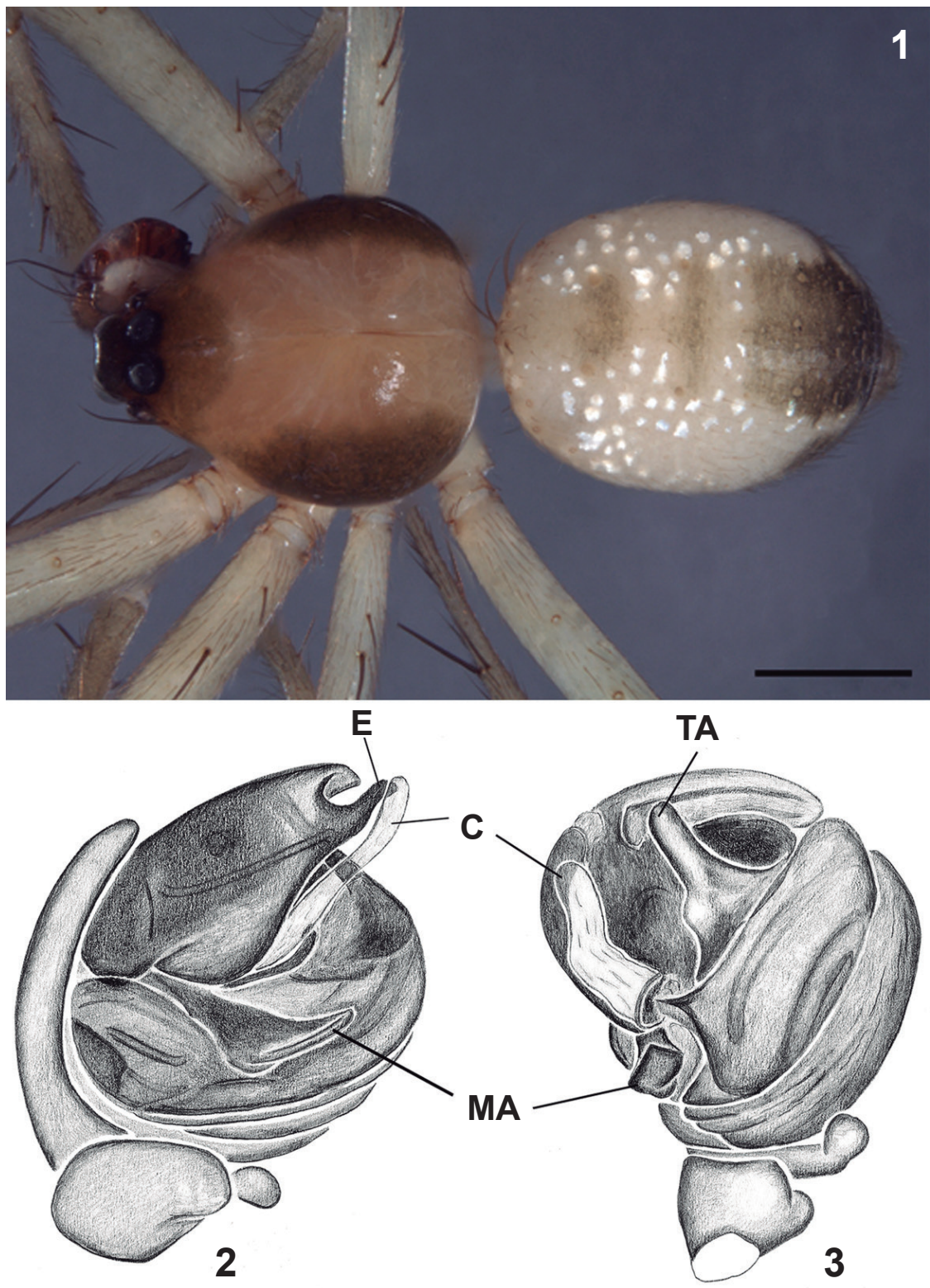

Figs 1-3. Mangora brokopondo Levi, 2007, male: 1, Habitus dorsal; Palpus: 2, mesal; 3, ventral (C, conductor; E, embolus; MA, median apophysis; TA, terminal apophysis). Scale bars: Fig. 1: $0.5 \mathrm{~mm}$; Figs 2, 3: $0.25 \mathrm{~mm}$. 

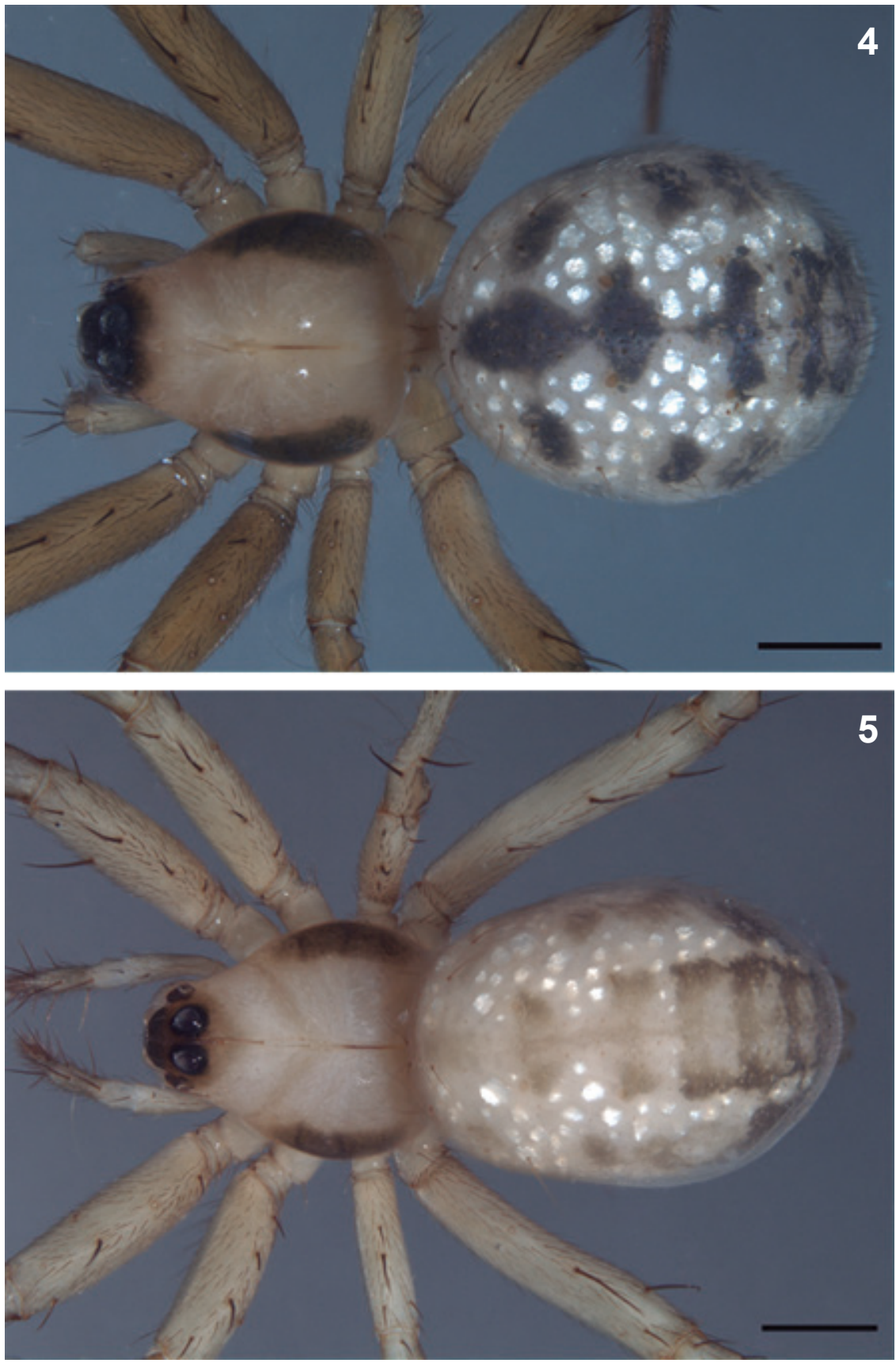

Figs 4-5. Mangora brokopondo Levi, 2007, female: habitus dorsal, variation in coloration pattern. Scale bars: $0.5 \mathrm{~mm}$.

AME 0.1, ALE 0.06, PME 0.09, PLE 0.07; AME-ALE 0.05, PME-PLE 0.08, AME-AME 0.08, PME-PME 0.07. Eyes with black borders. Carapace dark yellow, with brown band on each side of thoracic region, cephalic region brown (Fig. 1). Endites and labium gray. Sternum, chelicerae and legs yellowish, slightly gray. Metatarsus and tarsus brownish. Abdomen longer than wide, cylindrical. Dorsal side light yellow, border black, dark median strip anteriorly interrupted by many white dots (Fig. 1). Ventral side light gray, epigastric area slightly darker.

Material examined. BRAZIL, Pará: Melgaço (Caxiuanã, [143'59.2”S, 51³0’38.6”W]), , 11.VIII.1996, A. A Lise col. (MCTP 9376); (Floresta Nacional de Caxiuanã, Estação Científica Ferreira Penna, 143'59.2”S, 51³0’38.6”W), ㅇ, 22.IV.2006, R. A. Oliveira col. (MPEG 19086); (0144'15.5”S, 5126'42.0”'W), q, 11-20. VII.1998, B. Silva col. (MPEG 9444); Oे, 05.IX.1999, B. Mascarenhas col. (MPEG 9454); 1,2002, A. B. Bonaldo et al. col. (MPEG 19084);

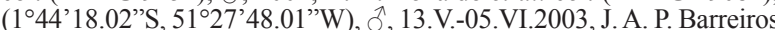
col. (MPEG 19085); (144'60.3”S, 51 27'27.9”'W), ${ }^{\circ}$, no date (MPEG 19087); Belterra (41 km S, Flona Tapajós, $3^{\circ} 1^{\prime} 0.05^{\prime}$ 'S, 5457'49.82”W), §, 30.IX.2008, N. Abrahim col. (MPEG 19769).

Variation. Females of $M$. brokopondo present considerable variation in the abdominal color pattern. LEVI (2007) illustrated one female with white pigment spots and contrasting black patches on the dorsum of the abdomen. Some of the females from FLONA Caxiuanã present the same abdominal pattern (Fig. 4, MPEG 9444) described by Levi, whereas others present a different arrangement of color patches, which are lighter and 
less contrasting, as illustrated in Fig. 5 (MPEG 19086). These findings suggest that the color pattern is not much informative for recognition of this species, as previously indicated by LEVI (2007).

Distribution. Brazil (state of Pará), Guyana and Suriname.

\section{Mangora hirtipes (Taczanowski, 1878)}

(Fig. 6)

Epeira hirtipes TACZANOWSKI, 1878:164, pl. 2, fig. 15 ( 9 lectotype and $\Phi$ paralectotype designed by Levi (2007) from Amable María [Dept. Junín, Prov. Tarma, 640 m. on Río Chanchamayo], Peru, ca. 1870s, deposited in Polish Academy of Science, not examined). Mangora hirtipes: Levi, 1991:177; 2007:124, figs 563-567, map 5E.

Mangora woytkowskii LEVI, 2007:133, figs 603-604 (ð holotype from Divisoria, 1,400 m, Húanuco, Peru, F. Woytkowski col., 23.IX.03.X.1946, deposited in American Museum National History, not examined). Syn. n.

Note. Males and females were collected together in many localities in the state of Pará: FLONA Caxiuanã, Itaituba and Juruti municipalities. LEVI (2007) described M. woytkowskii based on the holotype from Peru and additional material from Jacareacanga, Pará, Brazil. In this same revision, Levi redescribed the female of $M$. hirtipes and listed material from FLONA Caxiuanã, where several males and females were recently collected together. Although we have not examined the types, the drawings by LEVI (2007) and the examination of some females identified by him (MCP 9317 and MCP 9381) made it clear that these names are synonyms.

Material examined. BRAZIL, Pará: Melgaço (Caxiuanã, [142’24”S, 51²7’34.3”W]), 2 ㅇ, 11.VIII.1996, A. Lise et al. col. (MCTP 9381); ᄋ, 06-16.VIII.1996 (MCTP 9317); (Floresta Nacional de Caxiuanã, Estação Científica Ferreira Penna, 142’24”'S, $51^{\circ} 27^{\prime} 34.3 ” \mathrm{~W}$ ), O 19.IV.2006, C. A. Souza col. (MPEG 19097); ㅇ, 01.X.2005, N. Abrahim col. (MPEG 19107); (01²4'21.6”'S,

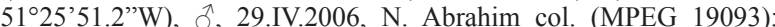
q, 8.X.2005, J. A. P. Barreiros col. (MPEG 19098); ㅇ, C. A. Lopes col. (MPEG 19110); ㅇ, 24.IV.2006, E. J. Sales col. (MPEG 19108); (0143'43.2”S, 51²9'07'W), +, 21.V.2006, J. A. P. Barreiros col (MPEG 19103); ( (MPEG 19109); ㅇ, 28.IX.-3.X.2005 (MPEG 19112); (0143’59.2”'S, 51³0’38.6”W), 9, 22.IV.2006, J. A. P. Barreiros col. (MPEG 19094); + , 04.X.2003 (MPEG 19100); +, 17.IV.2006, R. B. Lopes col. (MPEG 19096); 9 , C. A. Souza col. (MPEG 19099); 2 9, 29.IX.2005, C. A. Lopes col. (MPEG 19104); (0144'15.5”'S, $51^{\circ} 26^{\prime} 42.0^{\prime \prime} \mathrm{W}$ ),, , 05.VI.2004, A. B. Bonaldo col. (MPEG 9430); 3 , 13.II.2002 (MPEG 9470); ㅇ, no date, J. A. P. Barreiros col. (MPEG 19113);, , no date, no collector (MPEG 9539); $ᄋ$, no date, no collector

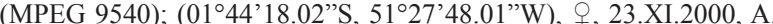

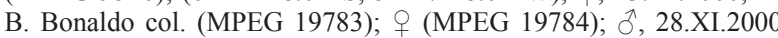
(MPEG 19776); $O$ (MPEG 19780); $O$ (MPEG 19782); O 03.XII. 2000 (MPEG 19781); ㅇ, 25.III.2002 (MPEG 19778); ㅇ, 13.V-5.VI.2003, J. A. P. Barreiros col. (MPEG 19111); 9 , 17.X.2003 (MPEG 19779); $\left(01^{\circ} 45^{\prime} 12.8^{\prime \prime S}\right.$, 51³1'14.7’W), +, 07.X.2005, C. A. Lopes col. (MPEG 19095); ㅇ, 23.IV.2006, E. J. Sales col. (MPEG 19105); (MPEG 18894), 12.X.2005, J. A. P. Barreiros col.; 9 (MPEG 19102); +, 23.IV.2006, R. B. Lopes col. (MPEG 19106); Portel (Floresta Nacional de Caxiuanã, Igarapé Caquajó, Plote PPbio, 0157'38.9”S,

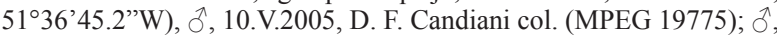
12.V.2005, J. A. P. Barreiros col. (MPEG 19777); Juruti ([29’23.21”S, 56 5'15.81”W], Ramal Pacoval, km 2), ㅇ, 21.XI.2007, N. F. LoMan-Hung \& D. F. Candiani col. (MPEG 19793); (Sítio Barroso, $02^{\circ} 27^{\prime} 41.7$ 'S, 56 00’11.6”'W), ㅇ, 14.VIII.2006, N. F. Lo-Man-Hung col. (MPEG 8229); , 16.XI.2007, E. S. Santos col. (MPEG 19792); 23.V.2009, N. C. Bastos col. (MPEG 19797); (Capiranga, $2^{\circ} 28^{\prime} 0.60 ” S$, $56^{\circ} 12^{\prime} 42.20^{\prime \prime}$ ), ô, 6.IX.2002, A. B. Bonaldo col. (MPEG 19794);
(MPEG 19808); ㅇ, 12.IX.2002 (MPEG 19795); 오, 5.IX.2002, D. D. Guimarães col. (MPEG 19789); + , 09.IX.2002 (MPEG 19791) ㅇ (MPEG 19796); 오, 14.IX.2002 (MPEG 19806); (02²8’22.1”'S, 56²'29.4”W), +, 18.XI.2007, C. M. Souza col. (MPEG 19773) (Capiranga, Linha 168e), , 11.III.2006, D. R. Santos-Souza col. (MPEG 9131); (Capiranga, Piçarreira), ㅇ, 19.XI.2007, D. F. Candiani col. (MPEG 19774); (Beneficiamento, 02³0'08.9”'S, 56 09'48.8”W), +, 8.V.2010, B. V. B. Rodrigues, N. C. Bastos \& N. F. Lo-Man-Hung col. (MPEG 19772); (02³0’27.4”S, 56¹0’39.5”W), ᄋ, 12.V.2010, N F. Lo-Man-Hung col. (MPEG 19798); , 10.VII.2010, N. Abrahim col. (MPEG 19787); ㅇ, 10.VIII.2010, B. V. B. Rodrigues col. (MPEG 19803); ㅇ, 22.VIII.2011, E. G. Cafofo col. (MPEG 19785); (Mutum, $\left.02^{\circ} 33^{\prime} 04.8^{\prime \prime S}, 56^{\circ} 13^{\prime} 32.5^{\prime \prime} \mathrm{W}\right)$, ㅇ, 20.XI.2007, N. F. Lo-Man-Hung col. (MPEG 19788); , 02.V.2010, B. V. B. Rodrigues col. (MPEG 19790); (02³3’06.9”'S, 56¹3’29.0”W), + , 3-8.VIII.2010, B. V. B. Rodrigues, N. C. Bastos \& N. Abrahim col. (MPEG 19807); ㅇ, 11.VIII.2010, N. Abrahim col. (MPEG 19799); 9 (MPEG 19804); , B.V. B. Rodrigues col. (MPEG 19801); ㅇ, 12.VIII.2010 (MPEG 19810); (02³6'11.2”S, 56¹2’36.3”W), ㅇ, 4.VIII.2004, D. R. Santos-

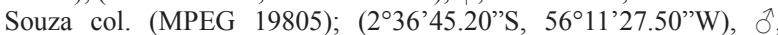
26.V.2009, N. F. Lo-Man-Hung col. (MPEG 19786); $\precsim$, 27.V.2009, N. Abrahim col. (MPEG 19800); + , 6.I.2012, N. C. Bastos col. (MPEG 19802); Itaituba (Rabelo, km 276 S Transamazônica, 5³4'20.06”S, 57¹8'24.34”W), Ô, 30.X.2009, E. G. Cafofo col. (MPEG 19771) స. 1.XI.2009 (MPEG 19770); Novo Progresso (Campo de Provas Brigadeiro Velloso, Serra do Cachimbo, mata torre, 09 ${ }^{\circ} 16^{\prime} 18.6^{\prime \prime} \mathrm{S}$, 54'56'22.9”W), Oै, 15.IX.2003, D. R. Santos-Souza col. (MPEG 6392); ㅇ, A. B. Bonaldo col. (MPEG 6077).

Distribution. Brazil (states of Acre, Amazonas and Pará), Guyana and Peru.

\section{Mangora alinahui Levi, 2007}

(Fig. 6)

Mangora alinahui LEVI, 2007:129, figs 590-594 ( holotype from Alinahui, $450 \mathrm{~m}, 20 \mathrm{~km}$ E Puero Napo, $01^{\circ} 00^{\prime} \mathrm{N}, 7^{\circ} 25^{\prime} \mathrm{W}$, Napo, Ecuador, E. S. Ross col., XI-XII. 1995, in California Academy of Sciences, not examined).

New record. BRAZIL, Pará: Bragança (1²'4.00”'S, $46^{\circ} 45^{\prime} 48.00^{\prime}$ 'W, Mata do Lobão), , 24.VI.2005, N. F. LoMan-Hung col. (MPEG 11411).

Distribution. Previously known from Ecuador, Bolivia, and the state of Amazonas, Brazil. Recorded here also from the state of Pará, Brazil.

\section{Mangora pia Chamberlim \& Ivie, 1936}

(Fig. 6)

Mangora pia Chamberlin \& Ivie, 1936:58, pl. 12, fig. 112 ( $q$ holotype from Barro Colorado Island, Panama, deposited in American Museum National History, not examined). Levi, 2007:134, figs 611-615.

Mangora belligerens Chamberlin \& Ivie, 1936:60, pl. 12, fig. 113 ( 3 holotype from Barro Colorado Island, Panama, deposited in American Museum National History, not examined). First synonymized by CHICKERING, 1954.

Mangora wiedenmeyeri SCHENCKel, 1953:18, fig. 15 (ㅇ holotype from El Pozón, Falcón, Venezuela, deposited in Naturhistorisches Museum, Basel, not examined). First synonymized by Levi, 2005.

New records. BRAZIL, Pará: Santarém (Alter do

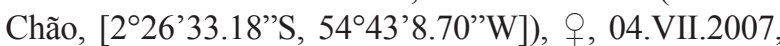
B. J. F. Silva col. (MPEG 16477); $\partial^{\lambda}$, + , 11.VII.2007 (MPEG 16469); 2 ㅇ (MPEG 16479); ㅇ, 15.VII.2007 (MPEG 16470); $9,04$. VII.2007, E. Borari col. (MPEG 


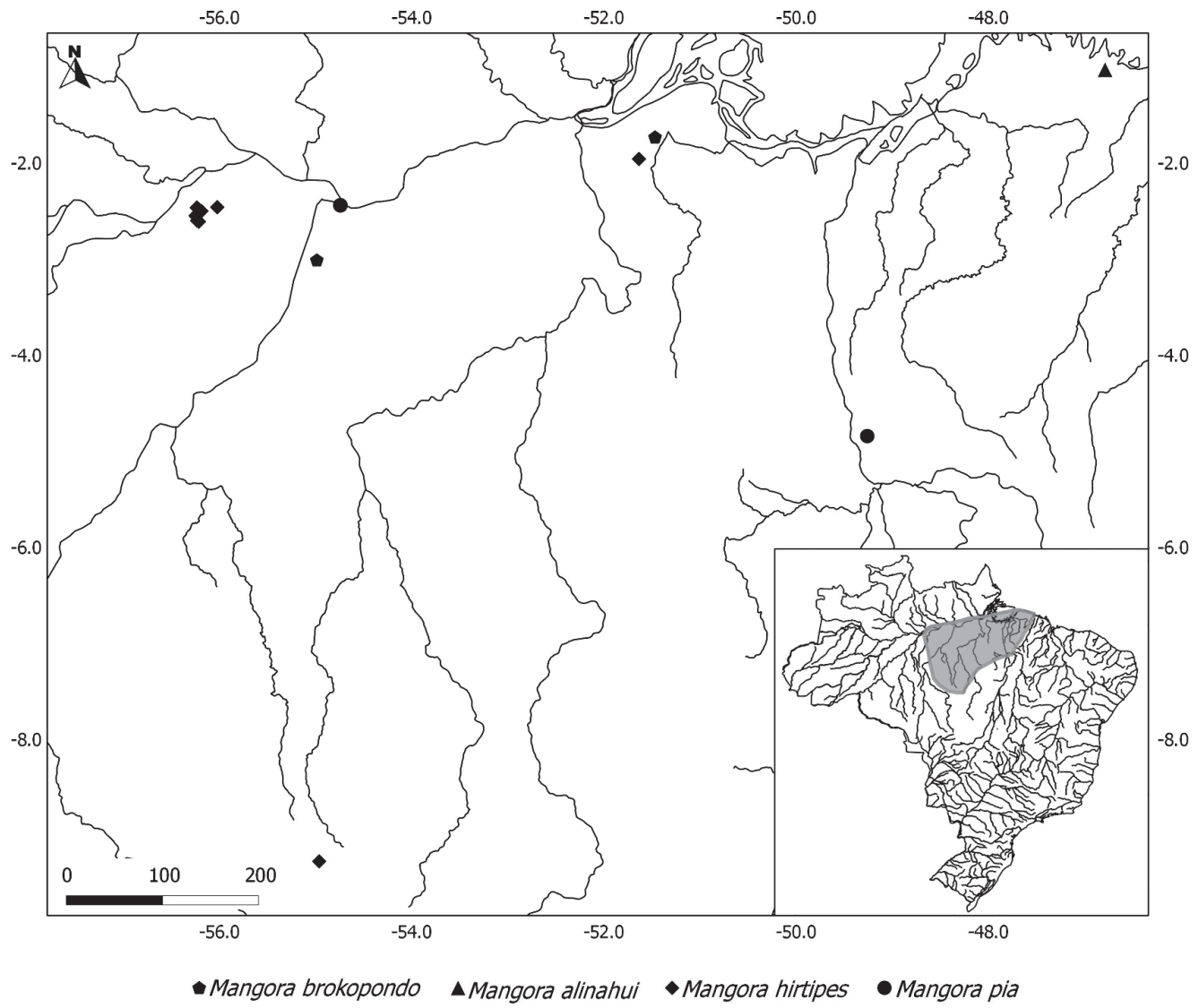

Fig 6. Distribution map of Mangora species in Oriental Brazilian Amazon.

16471); ふૈ, ๆ, 11.VI.2007 (MPEG 16467); ㅇ, 02.VII.2007, M. L. N. Sirotheau col. (MPEG 16476); , 15.VI.2007, R. R. dos Santos col. (MPEG 16465); §̃, 18.VI.2007, E. Borari col. (MPEG 16226); $\hat{\jmath}$, + (MPEG 16466); + , R. R. dos Santos col. (MPEG 16464); § (MPEG 16475); (Caratinga), 9 , 27-30.VI.2007, E. Borari col. (MPEG 16473); + (MPEG 16462); +, M. L. N. Sirotheau col. (MPEG 16463); (São Raimundo), ô, 11.VII.2007 (MPEG 16227); ㅇ (MPEG 16474); (Seringa), ㅇ 13.VII.2007 (MPEG 16228); , B. J. F. Silva col. (MPEG 16468); (Capiranga), ô, 15.VII.2007, M. L. N. Sirotheau col. (MPEG 16478); (Mocoto), +, 27-30.VI.2007, E. Borari col. (MPEG 16480); (Paraíso), ๆ, 14.VII.2007, M. L. N. Sirotheau col. (MPEG 16229); (São Luiz), , 02.VII.2007 (MPEG 16472); Nova Ipixuna (Fazenda Bom Retiro), §,, 17.IV.2012, M. B. Aguiar Neto col. (MPEG 19942); 우 (MPEG 19943); đ̊ (MPEG 19944).

Distribution. Previously known from Panama, Venezuela, Colombia and Brazil (state of Roraima). Recorded here also from the state of Pará, Brazil.

Acknowledgements. To Gustavo Ruiz for helpful suggestions on the illustrations; Nayane Bastos help sorting material and Aline Alves databased some of the records. The authors are supported by grants: ABB - PQ grant \#307463/2009-5, EGC \#382042/2011-5; MBAN \# 149264/2011-9, JR \#140646/2010-8, RS \#141884/20096 from CNPq; EGC \# Centro de Custo 78028002 from FUNAPE. Fieldwork opportunities and lab supplies were sponsored by "Núcleo Regional do Leste Paraense" (CNPq 558202/2009-8) from "Programa de Pesquisas em Biodiversidade - Amazônia Oriental" (PPBio). This article was partially produced during the discipline "Aracnologia" offered in 2011 by Alexandre Bonaldo and Gustavo Ruiz at the PostGraduation Program in Zoology/ Universidade Federal do Pará and Museu Paraense Emílio Goeldi, Belém, Pará, Brazil.

\section{REFERENCES}

Bonaldo, A. B.; Carvalho, L. S.; Pinto-da-Rocha, R.; Tourinho, A.; Miglio, L. T.; Candianni, D. F.; Lo-Man-Hung, N. F.; Abrahim, N.; Rodrigues, B. V. B.; Brescovit, A. D.; Saturnino, R.; Bastos, N. C.; Dias, S. C.; Silva, B. J. F.; Pereira-Filho, J. M. B.; Rhems, C. A.; Lucas, S. M.; Polotow, D.; Ruiz, G. \& Indicatti, R. 2009. Inventário e história natural dos aracnídeos da Floresta Nacional de Caxiuanã. In: LisBoA, P. L. B. org. Caxiuanã: desafios para a conservação de uma Floresta Nacional na Amazônia. Belém, Museu Paraense Emílio Goeldi, p.577-621.

Chamberlin, R. V. \& Ivie, W. 1936. New spiders from Mexico and Panama. Bulletin of the University of Utah 27(5):1-103.

Chickering, A. M. 1954. The spider genus Mangora (Argiopidae) in Panama. Bulletin of the Museum of Comparative Zoology at Harvard 111(5): 195-215

Levi, H. W. 1976. The orb-weaver genera Verrucosa, Acanthepeira, Wagneriana, Acacesia, Wixia, Scoloderus and Alpaida north of Mexico. Bulletin of the Museum of Comparative Zoology at Harvard 147:351-391. 1988. The neotropical orb-weaving spiders of the genus Alpaida (Araneae: Araneidae). Bulletin of the Museum of Comparative Zoology at Harvard 151:365-487.

1991. The Neotropical and Mexican species of the orb-weaver genera Araneus, Dubiepeira, and Aculepeira (Araneae: Araneidae). Bulletin of the Museum of Comparative Zoology at Harvard 152:167-315.

1995. Orb-weaving spiders Actinosoma, Spilasma, Micrepeira, Pronous, and four new genera (Araneae: Araneidae). Bulletin of the Museum of Comparative Zoology at Harvard 154:153-213. 2005. The orb-weaver genus Mangora of Mexico, Central 
America, and the West Indies (Araneae: Araneidae). Bulletin of the Museum of Comparative Zoology at Harvard 158(4):139-182.

2007. The orb weaver genus Mangora in South America (Araneae, Araneidae). Bulletin of the Museum of Comparative Zoology at Harvard 159:1-144.

PlatNICK, N. I. 2013. The world spider catalog, version 13.5. American
Museum of Natural History. Available at: $<$ http://research.amnh.org/ $\mathrm{iz} / \mathrm{spiders} / \mathrm{catalog}>$. Accessed on 14.02.2013.

SCHENKEL, E. 1953. Chinesische Arachnoidea aus dem Museum HoanghoPeiho in Tientsin. Boletim do Museu Nacional, Zoologia 119:1-108.

Taczanowski, L. 1878. Les Aranéides du Pérou central. Horae Societatis Entomologicae Rossicae 14:140-175. 